\title{
Case detection, gender and disability in leprosy in Bangladesh: a trend analysis
}

\author{
J. H. RICHARDUS*, A. MEIMA*, R. P. CROFT** \& \\ J. D. F. HABBEMA* \\ *Department of Public Health, Erasmus University Rotterdam, \\ The Netherlands \\ **The Danish-Bangladesh Leprosy Mission, Nilphamari, Bangladesh
}

\author{
Accepted for publication 14 January 1999
}

Summary A trend analysis is presented of all newly detected leprosy cases over an 18-year period (1979-1996) in a highly leprosy endemic area of Bangladesh. A total of 23,678 new cases were registered, with an average of 860 new cases per year in the first 12 years, and increasing to around 3000 in 1996. The male : female (M:F) ratio decreased from $2 \cdot 3$ to $1 \cdot 4$. The proportions of newly detected cases with MB leprosy and of newly detected cases with any disability decreased over time. These reductions were more marked in the higher age groups of both sexes. The reduction in disability was primarily attributable to a decline in grade 2 disability. New case detection rates (NCDR) of all leprosy patients per 10,000 general population increased for males from 3 to 6; and for females from 1 to 4, while the NCDR of MB leprosy decreased in males from $1 \cdot 4$ to $0 \cdot 6$, and in females fluctuated around $0 \cdot 45$. The NCDRs of leprosy patients with disabilities showed an initial decrease in the first period, especially in males, but later showed an increase. The NCDR of males with disability was about twice as high as that of females. Finally, female NCDRs in the ages between 15 and 30 were low by comparison with the male NCDRs at the same time. This may be due to the sociocultural characteristics of the Bangladeshi society, with gender differences in exposure, health seeking behaviour and opportunities for case detection. Operational changes in the control programme have contributed to the changed profile of newly detected cases. This study shows that the application of general population statistics is essential for understanding the dynamics in leprosy control programmes under changing operational conditions. Combining case detection figures with such statistics helps to identify population groups that are possibly not benefiting sufficiently from the services provided, and to clarify the dynamics in control programmes and the future trends and programme requirements.

Correspondence to: Jan Hendrik Richardus, Department of Public Health, Erasmus University Rotterdam, PO Box 1738, 3000 DR Rotterdam, The Netherlands. Tel: +31 10 4088285, Fax: +31 10 4089449, e-mail: richardus@mgz.fgg.eur.nl 


\section{Introduction}

Interest in gender inequality is increasing in public health research. Many health status indices show females to be disadvantaged. Males and females have different health risks due to differences in biology, but also due to differences in their social roles and expectations. Women may have different exposure to disease and infection, and diseases may have different effects on women, not only medically, but also in sociocultural and economical aspects. This is particularly marked in the developing world where women may also have less access to health services than men. In a disease with a strong social stigma, such as leprosy, the impact of gender inequality may be considerable. A recent review on women and leprosy ${ }^{1}$ concluded that sex differences in case detection of leprosy exist, but information varies considerably between country. The relative importance of biological, health services-related and community-related factors is also unclear and differs between region or country. More information is needed on gender issues, both for understanding the epidemiology of leprosy and for taking operational measures in leprosy control programmes to reduce gender inequalities in health.

This study presents a trend analysis of newly detected leprosy patients over an 18-year period (1979-1996) in a moderate to high leprosy endemic area in North West Bangladesh. The objective was to investigate the relative contribution of females to newly detected cases in relationship to age, leprosy classification and disability, in varying operational circumstances. The time period under study includes three relatively distinct operational situations: the pre-multidrug therapy (MDT) era; a transition phase with the introduction of MDT; and the present era where the introduction of MDT has been completed and full coverage of the geographical area with leprosy control services has been achieved. A study of gender differences in new case detection rates in these distinct time periods may reveal the extent to which males and females are affected by leprosy, the effect of leprosy control services, and possibly illustrate reasons for gender inequalities.

\section{Materials and methods}

PROJECT DESCRIPTION

The study is based on data from the Danish Bangladesh Leprosy Mission (DBLM) in Nilphamari, Northwest Bangladesh. DBLM has conducted a vertical leprosy programme in a moderate to high endemic area (estimated prevalence in 1993: approximately 5 per 1000) since $1977 .^{2}$ The DBLM leprosy control programme covers the districts of Nilphamari, Rangpur, Thakurgaon and Panchagar. The population (1991 census) was 5,529,600 and the area covered is $7163 \mathrm{~km}^{2}$, giving a population density of 772 persons per $\mathrm{km}^{2}$. Since 1994, the DBLM has been responsible for leprosy control in the four districts of the Rajshahi Division. Soon after DBLM was established, the programme was treating more than $95 \%$ of registered leprosy patients in these districts.

TIME FRAME

An 18-year period (1979-1996) is included in this analysis. During this time frame, three distinct 6-year periods with different operational characteristics can be distinguished. In the first period (1979-1984), the project had started on a small scale, with a limited number of 
staff and clinics. Treatment was with dapsone monotherapy and case finding primarily passive. The second period (1985-1990) was a transition period. MDT was introduced for all new cases, the geographical coverage with clinics expanded within the four districts, and the programme became better known and accepted by the population. By 1991, MDT had been introduced completely in the programme. In the last period (1991-1996), major financial impetus was given to the programme and complete coverage of all districts with a network of easily accessible clinics was established, with more than 50 clinics and 110 paramedical workers involved. This period saw expansion into a comprehensive and accepted leprosy control project with health education programmes, rapid village surveys, regular follow-up of all patients, field treatment of reactions and nerve function impairment, hospital services for reconstructive surgery, and rehabilitation programmes. General health services (provided by the government) developed less rapidly, and BCG coverage remained low during this period.

\section{INCLUSION CRITERIA}

All cases diagnosed with leprosy for the first time and registered in the DBLM programme during the time period 1979-1996 are included in this study. They are presented in the age categories: $0-14$ years, $15-39$ years and $\geq 40$ years. It is estimated that $5 \%$ of patients come from outside the DBLM project area. They were not excluded because the home village of DBLM patients could not always be established accurately.

\section{LEPROSY CLASSIFICATION}

From the beginning of the DBLM programme the Ridley-Jopling classification was recorded for the type of leprosy of a new patient and skin smears were taken from most new patients. ${ }^{3-5}$ To prevent inconsistencies in trend descriptions caused by changing definitions over time, the following criteria were applied for data analysis: cases with Ridley-Jopling classification $\mathrm{BB}, \mathrm{BL}$ and $\mathrm{LL}$ and all cases with positive skin smears $(\mathrm{BI}>0)$ are defined as $\mathrm{MB}$, and all other cases are defined as PB.

\section{DISABILITY GRADING}

Disability is graded according to the WHO leprosy disability grading system. ${ }^{4}$ For hands and feet, grade 0 is defined as no anaesthesia, visible deformity or damage; grade 1 as anaesthesia present, but no visible deformity or damage; and grade 2 as visible deformity or damage. The WHO has defined a comparable system for grading disabilities of the eyes, also on a scale from 0 to 2 .

\section{POPULATION STATISTICS}

Detailed population statistics including sex and age distributions are available at district level in the Bangladesh census data of 1981 and 1991, allowing the calculation of population based case detection rates. ${ }^{6-8}$

\section{NEW CASE DETECTION NUMBERS AND RATES}

The newly detected leprosy cases in the 18-year period of this study are analysed at two 
levels. The first level only includes the newly detected cases (or numerator), and studies their distribution patterns over time according to sex, age, leprosy classification and disability. In the second level, the denominator (population from which the cases originate) is taken into account to calculate new case detection rates (NCDR).

\section{STATISTICAL ANALYSIS AND ILLUSTRATION OF TRENDS}

For comparison of proportions and case detection rates, the Chi-square test was used. Logistic regression was performed for testing the statistical significance of trends. Statistical analysis was carried out in SPSS. To illustrate trends over the study period, curves (smoothing splines) were fitted to several graphs with the statistical package S-Plus 4.

\section{Results}

Figure 1 shows the new cases detected over the 18-year period in absolute numbers according to sex, and gives male : female $(\mathrm{M}: \mathrm{F})$ ratios. Graphs are given for all ages combined and for the age groups 0-14 years, 15-39 years, and 40 years and above, separately. The total number of new leprosy patients registered over the whole period is 23,678 with an average of 860 new cases per year in the first 12 years, and increasing in the last 6-year period to around 3000 new patients in 1996 . The $\mathrm{M}: \mathrm{F}$ ratio decreased from approximately $2 \cdot 3$ in the first 6-year period to around 1.4 for 1994-1996. The age groups 15-39 and 40+ years are responsible for this decrease; in the children's age group ( $0-14$ years) the M:F ratio fluctuated between $1 \cdot 1$ and $2 \cdot 0$ without showing a clear trend.

Figure 2 gives the MB proportion, according to sex and presented in three age groups. The initial proportion of MB leprosy in 1979 in all age groups together was $58 \%$ for males and $45 \%$ for females. This proportion reduced over time to $10 \%$ for males and $4 \%$ for females in 1996. The reduction was most marked in the two higher age groups. In the child group, the proportion MB cases in boys fluctuated around 20\%, but fell in girls to less than 5\% in 1996. The declines took place mostly in the last 6-year period, as shown by the flow of the spline functions. The largest reduction in proportion of smear positives among newly detected cases took place in the last 6-year period (proportion smear positive among those with smear taken for the three subsequent 6-year periods: $29 \%, 23 \%$ and $9 \%$, smears were taken from at least 93\% of new cases).

Figures 3 and 4 show the proportion of new cases presenting with disabilities. Figure 3 shows the proportion with any disability (WHO grading 1 plus 2), according to sex and presented in the three age groups. There is a nearly parallel decline in proportion with disability in males and females. In males the overall decline is from $65 \%$ in 1979 to $20 \%$ in 1996; in females the decline is from $55 \%$ to $10 \%$. The decline is observed in all three age groups and is steeper for the two higher age groups, with an acceleration in the last 6-year period (as is illustrated by the flow of the spline functions). Figure 4 includes the proportion of new patients presenting with disability according to WHO grade 1, 2, and 1 plus 2, categorized by sex. It can be seen that the decline in grade 2 disability contributed most to the decline in total disability.

Figures 5-8 present new case detection rates per 10,000 population per year (NCDRs) for the DBLM project area. Total sex-wise NCDRs are given in Figure 5. The male NCDR increases from $2 \cdot 5$ per 10,000 in 1979 to nearly 6 per 10,000 in 1996, a more than two-fold 

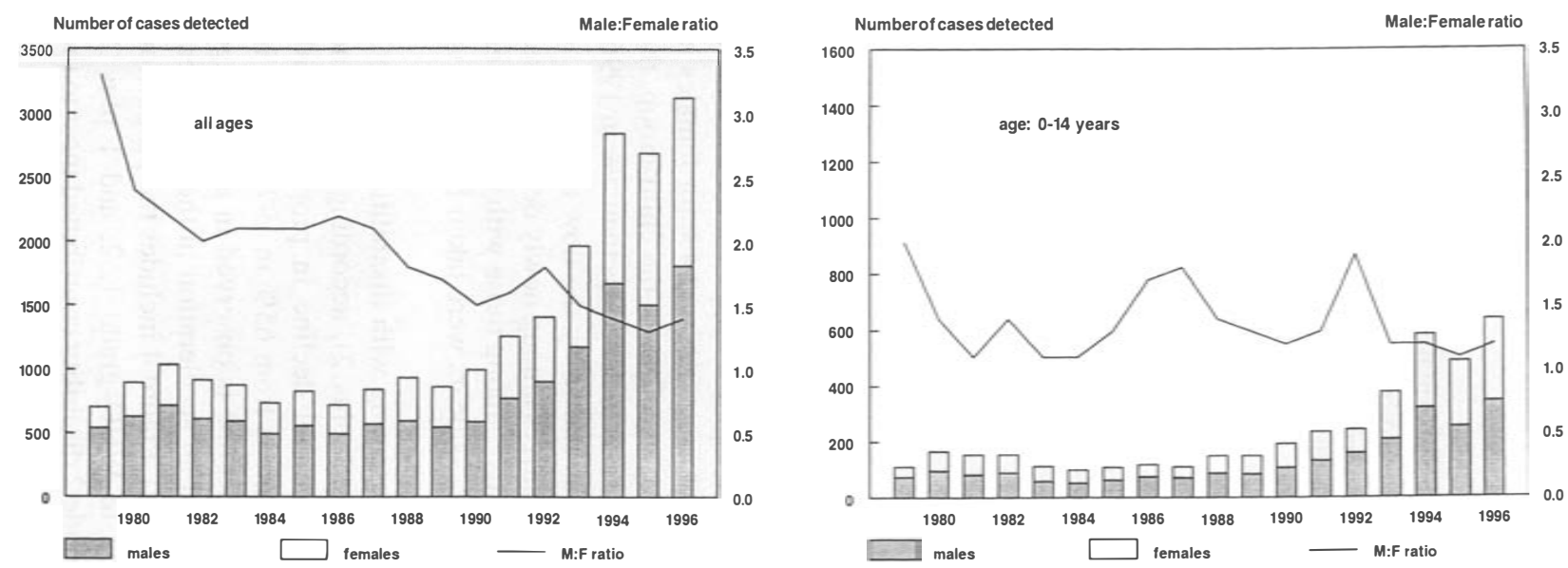

Number of cases detected

Male:Female ratio
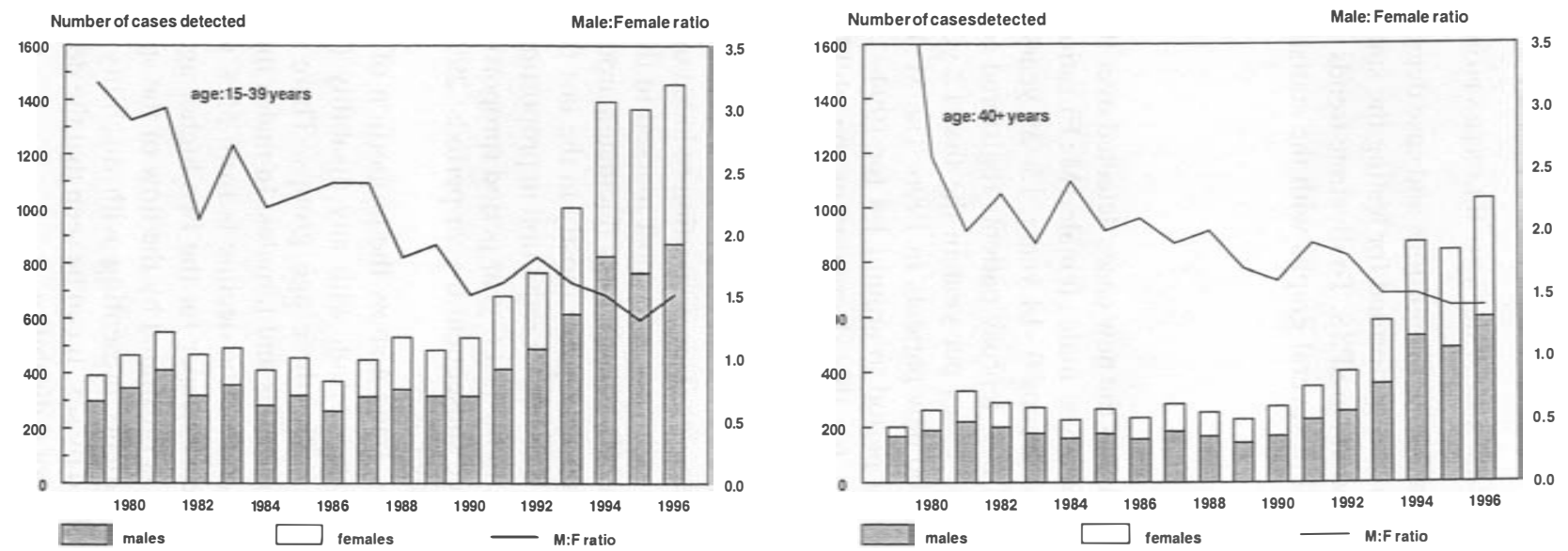

Figure 1. Number of newly detected leprosy cases from 1979 to 1996, according to sex and including male : female ratios, and presented in the following age groups: all ages; 0 14 years; $15-39$ years; and 40 years and above. 


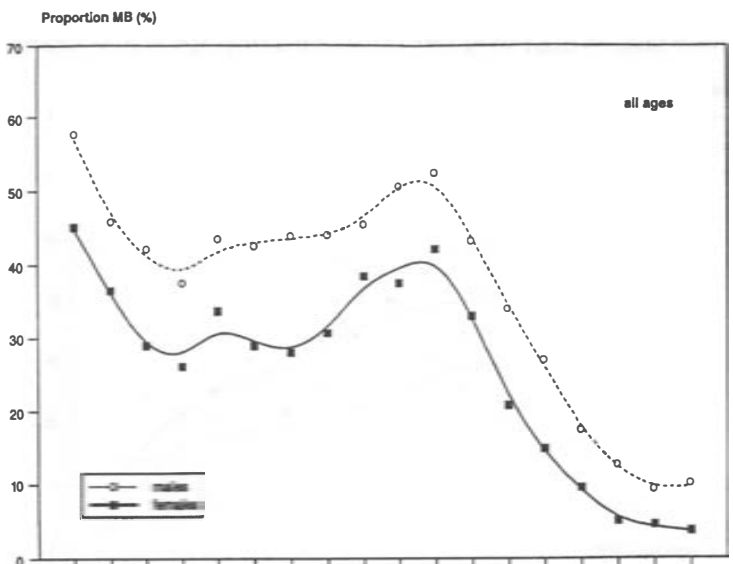

197919801981198218831984198519861987188819891980199119921993199419951996

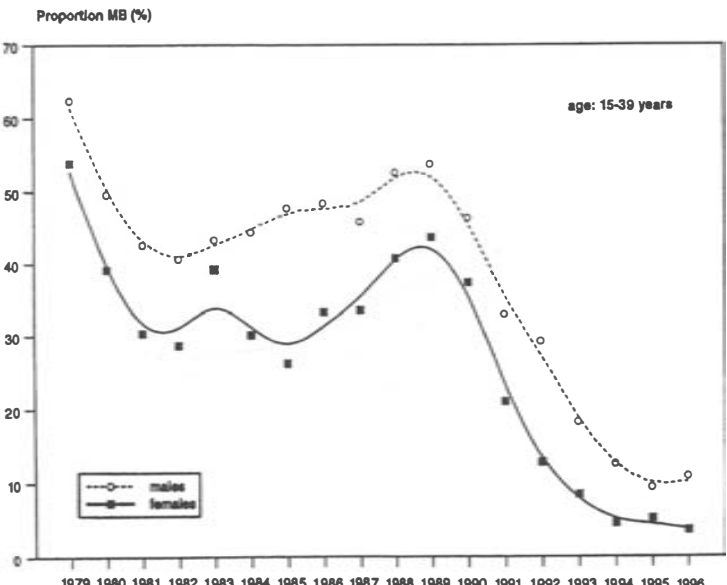

Proporton MB (x)

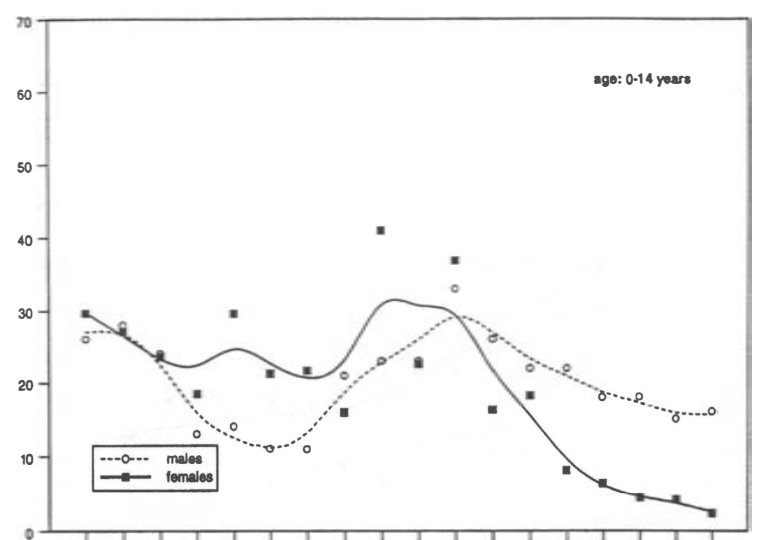

197919801981198219831984198519861987198819891990199119921899198419951996

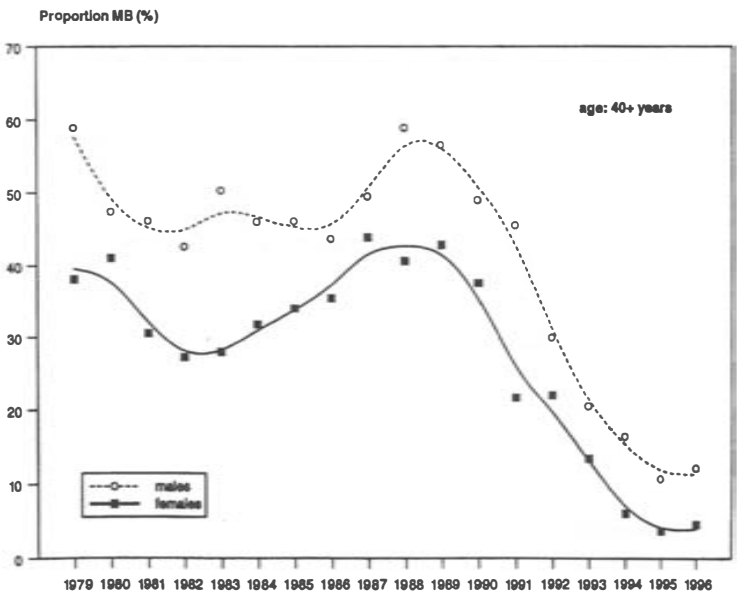

Figure 2. Proportion of newly detected leprosy cases from 1979 to 1996 with MB leprosy, according to sex and presented in the following age groups: all ages; 0-14 years; 15- 

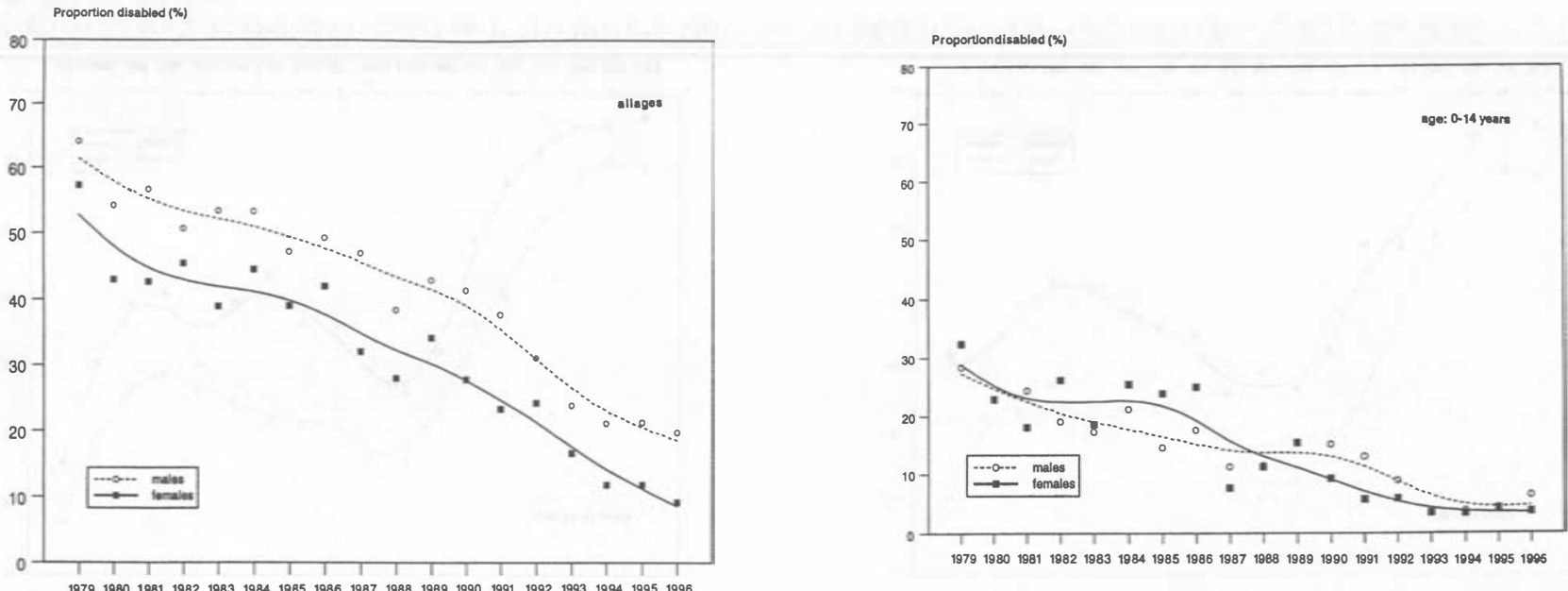

197910801881198219831984198519861987198819891990199119921993199419951996

197919801981198219831984198519861987198819891990199119921993199419951000
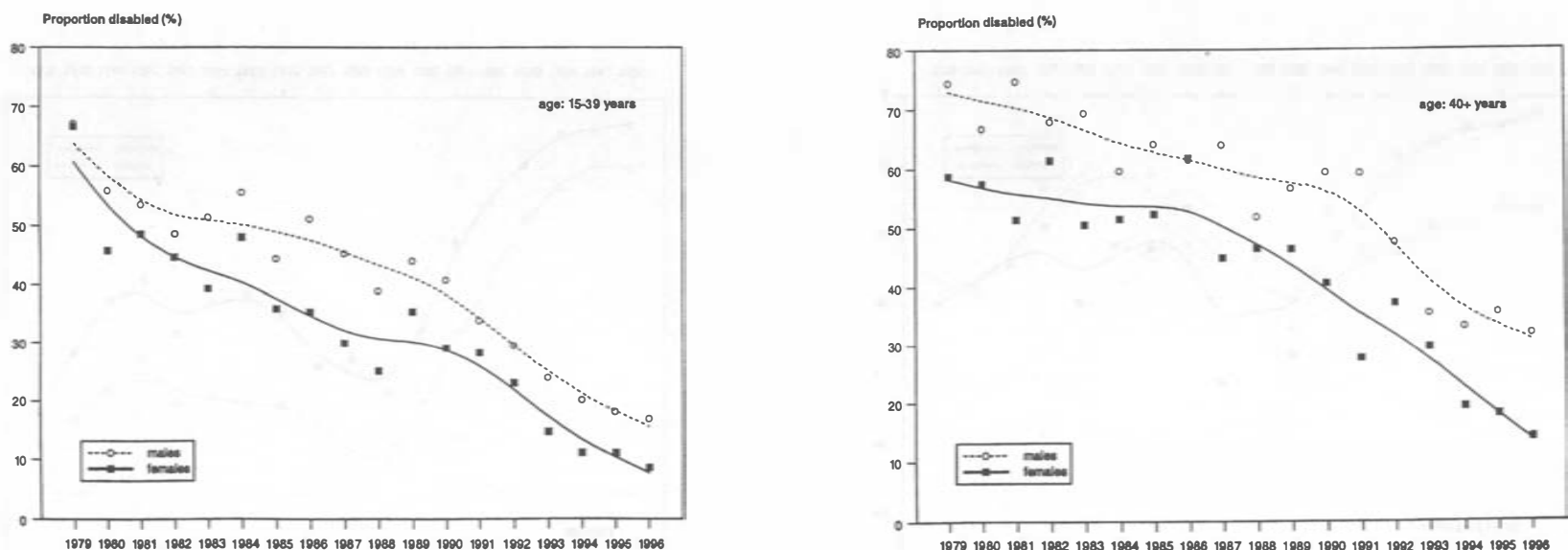

Figure 3. Proportion of newly detected leprosy cases from 1979 to 1996 with disability grade 1 or grade 2, according to sex and presented in the following age groups: all ages; 0-14 years; $15-39$ years; and 40 years and above. 
Proportion disabled (\%)

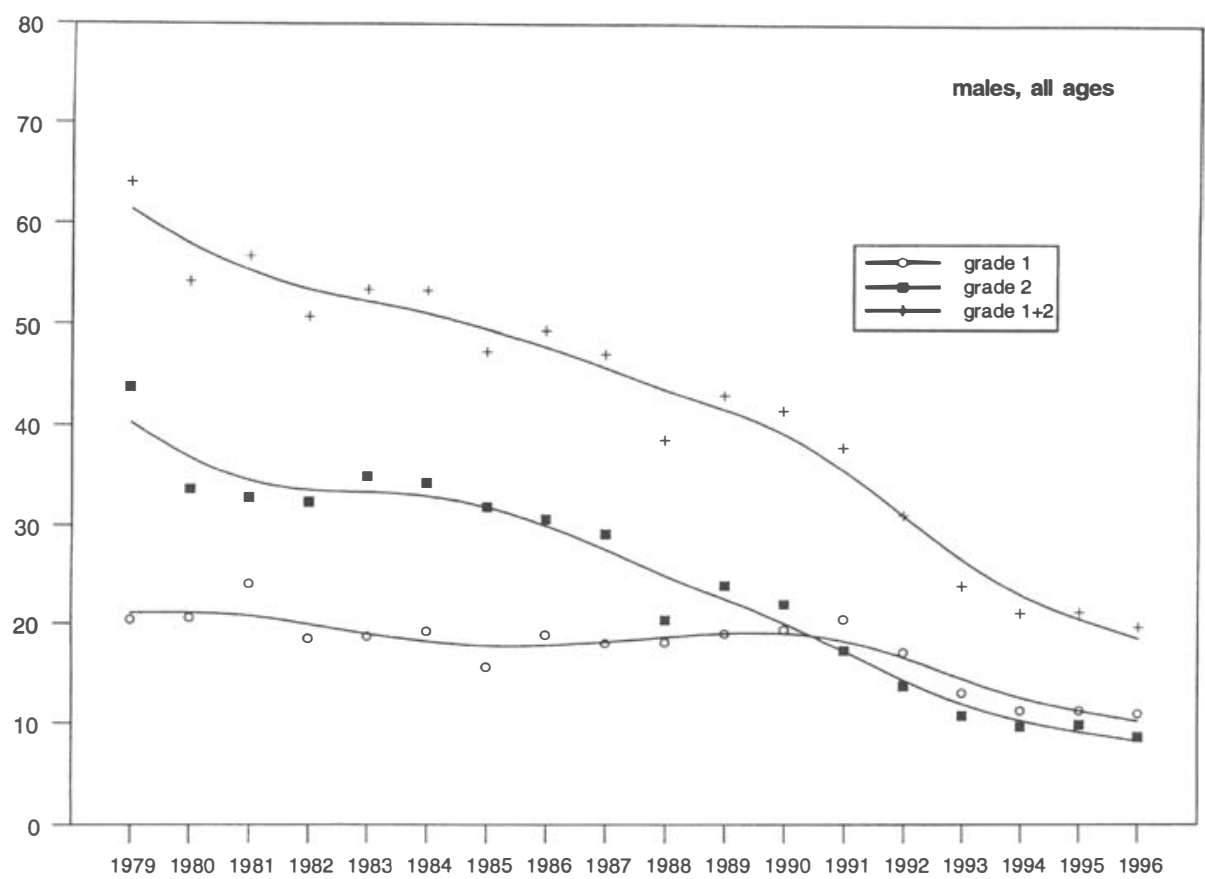

Proportion disabled (\%)

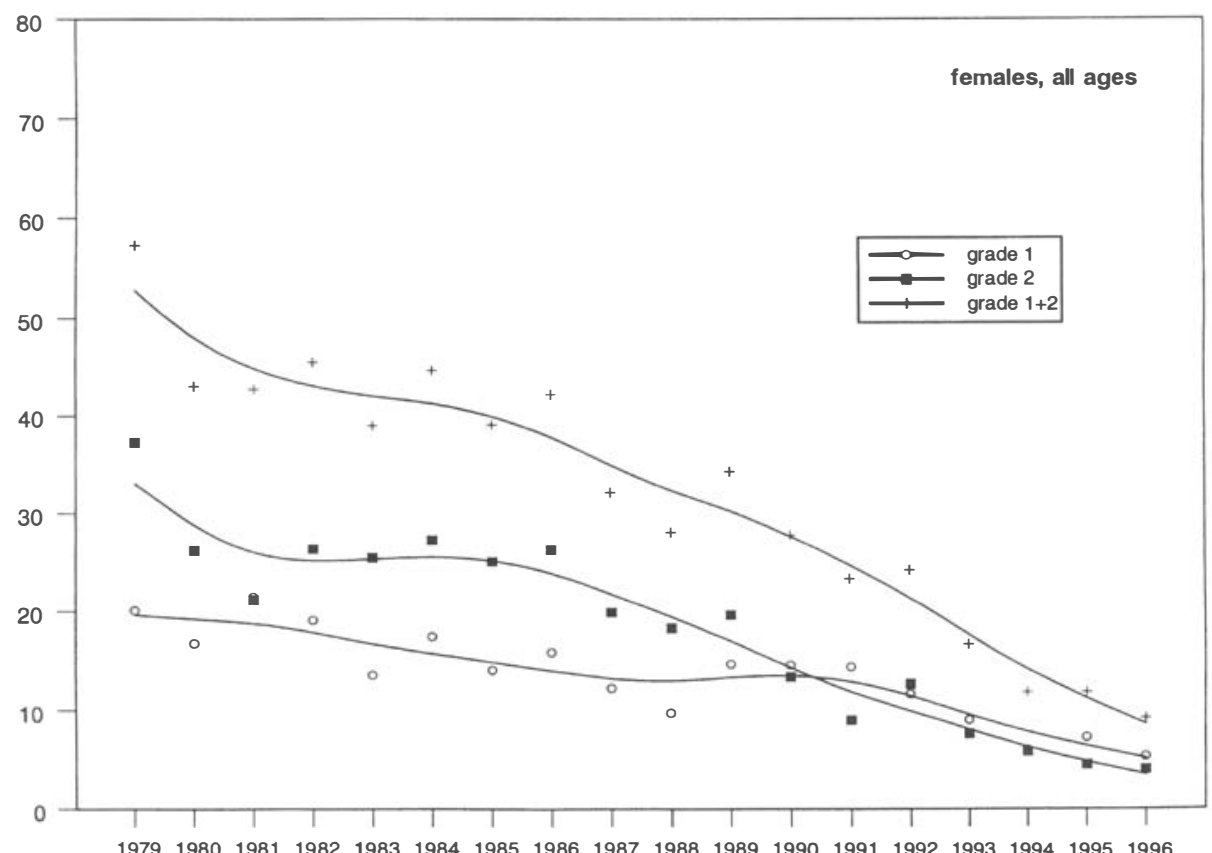

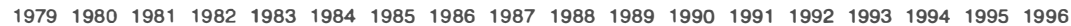


New case detection rate $/ 10,000 /$ year

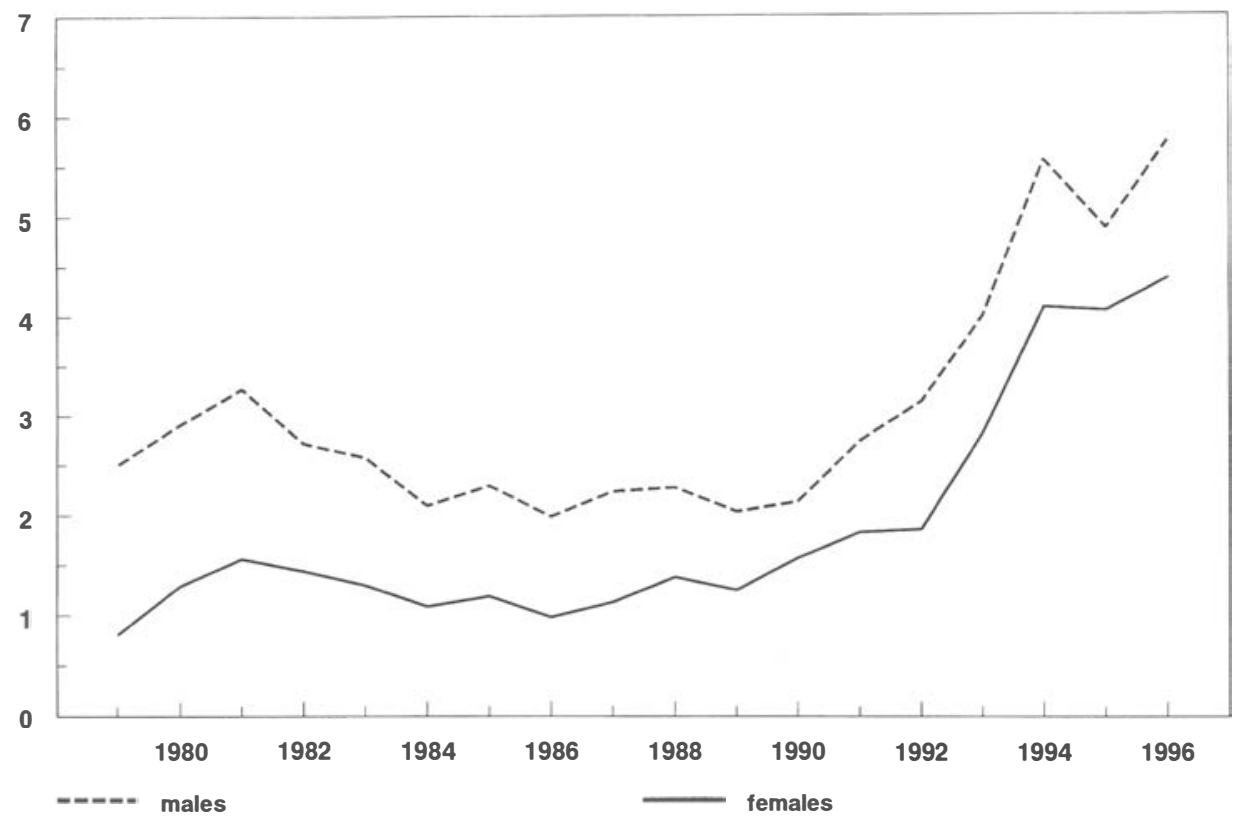

Figure 5. New case detection rate of leprosy patients in the DBLM project area per 10,000 general population per year from 1979 to 1996 , according to sex.

increase. A four-fold increase from approximately 1 to 4 per 10,000 is observed for females. In Figure 6, the age specific NCDRs are shown separately for males and females for each of the three 6-year periods. The shapes of the age specific NCDR curves for males and females are quite distinct at young adult ages. After an initial increase, the NCDR in females shows a marked decrease at age 15 , only to increase again after age 24 . In males the increase in NCDR continues into adulthood, with stabilisation setting in around age 30. This pattern is most marked in the last 6-year period. The NCDR increased considerably in all age groups for both males and females in the third 6-year period (1991-1996). Although the increase in NCDR varied with age, shifts in case detection towards young or old age from one period to the next, indicating changes in the age distribution of underlying incidence, could not be shown.

Figure 7 gives the NCDR for new patients with MB leprosy. The male NCDR per 10,000 decreased from around 1.4 in 1979 to 0.6 in 1996. The female NCDR for MB leprosy fluctuated between $0 \cdot 3$ and $0 \cdot 5$ per 10,000 in the first two periods, and started to decline in the third 6-year period, attaining a level of $0 \cdot 16$ per 10,000 in 1996. Finally, NCDRs of new patients with disability (grade 1 only, and grade 1 plus 2) are given in Figure 8. By comparison with females, NCDRs for males with disability are more than twice as high for both grade 1 disability and any disability (grade 1 plus 2). After decreasing or stable initial trends, the NCDRs for both males and females showed an increase after 1988 for all disability grades.

Figure 4. Proportion of newly detected leprosy cases (all ages) from 1979 to 1996 with disability grade 1; grade 2; and grade $1+2$, according to sex. 


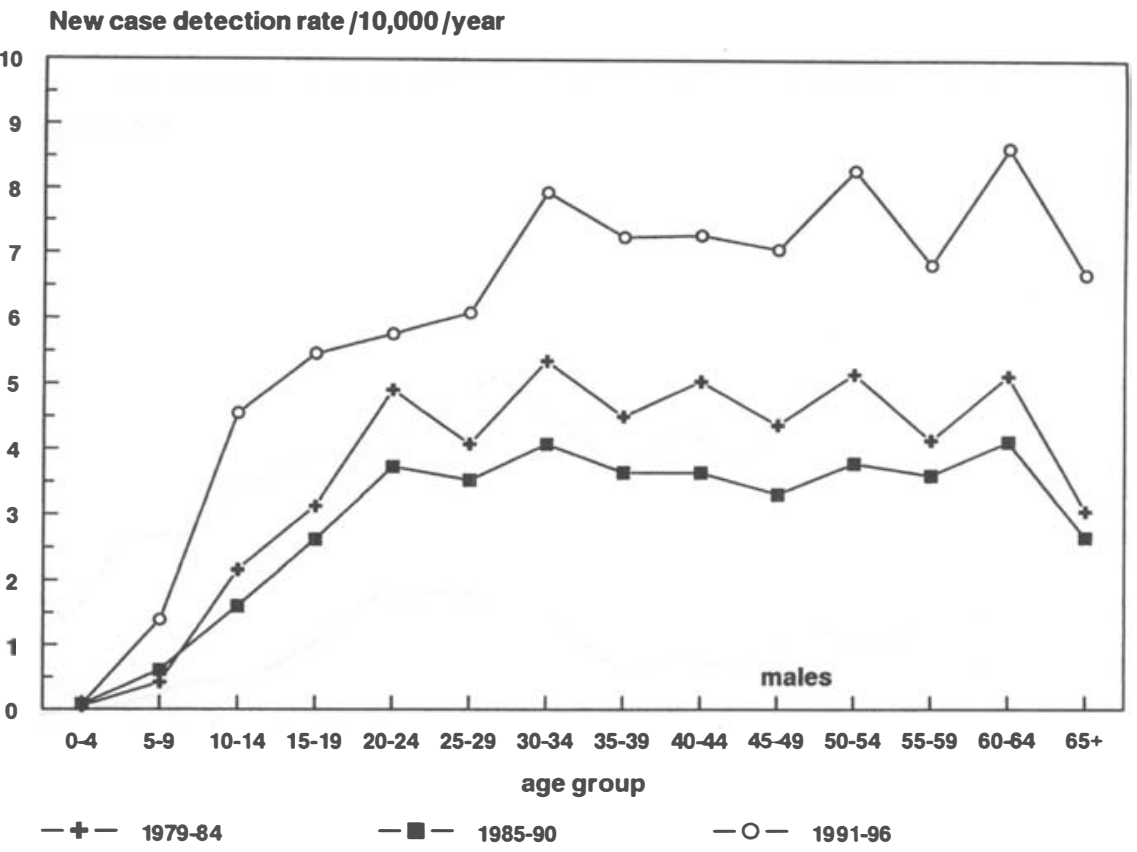

\section{New case detection rate $/ 10,000 /$ year}

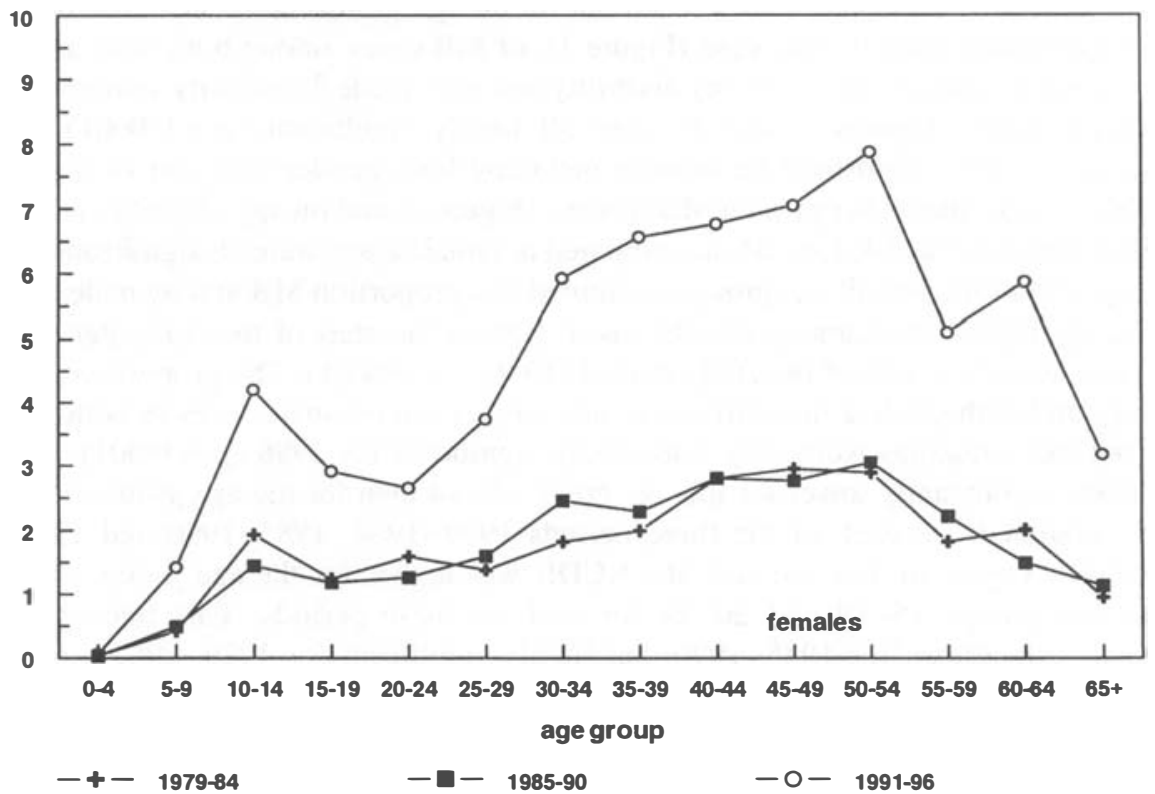

Figure 6. Age specific new case detection rates of leprosy patients in three time periods: 1979-1984; 1985-1990; 1991-1996, according to sex. 


\section{New case detection rate $/ 10,000 /$ year}

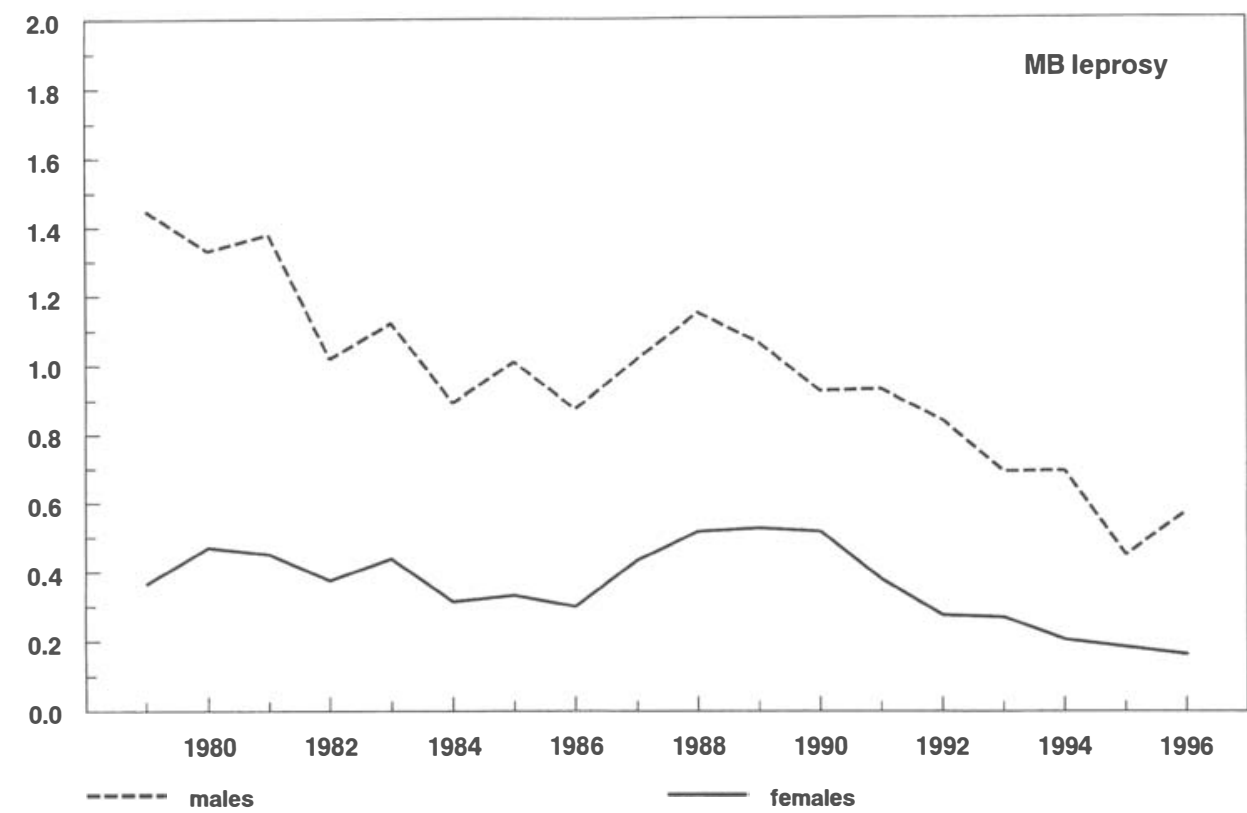

Figure 7. New case detection rate of MB leprosy patients in the DBLM project area per 10,000 general population from 1979 to 1996 , according to sex.

Statistical tests for trends were carried out for all age groups combined. The decreasing trends in the proportion of male case (Figure 1), of MB cases among both male and female cases (Figure 2), and of cases with any disability and with grade 2 disability among both male and female cases (Figures 3 and 4$)$ were all highly significant $(p<0.0001)$. Logistic regression was also performed for models including both gender and year of registration. For these models, the odds ratios on MB leprosy (Figure 2) and on any disability and grade 2 disability (Figures 3 and 4 ) for male as compared to female cases were all significantly greater than $1(p<0 \cdot 0001)$. For all age groups combined, the proportion MB among male cases was significantly higher than among female cases, both at the start of the study period (1979, $p<0.005)$ and at the end of the study period $(1996, p<0.0001)$. The proportions with any disability and with grade 2 disability were also higher among male cases in both 1979 and 1996 , but these findings were only statistically significant for $1996(p<0 \cdot 001)$. The male NCDR was significantly lower for the age group 10-14 than for the age groups 15-19 and 20-24 combined, for each of the three periods 1979-1984, 1985-1990 and 1991-1996 $(p<0.0001$, Figure 6). For females, the NCDR was higher for the age group 10-14 than for the age groups 15-19 and 20-24 for each of these periods. This finding was not statistically significant for 1985-1990, but highly significant for 1979-1984, $(p<0 \cdot 001)$ and 1991-1996 $(p<0 \cdot 0001)$. Logistic regression showed a decreasing trend in NCDR for MB leprosy which was highly significant for both males and females $(p<0 \cdot 0001$, Figure 7). A statistically significant increasing trend in NCDR for leprosy with any disability was observed from 1988 onwards for males $(p<0.0001)$ but not for females $(p=0 \cdot 27$, Figure 8). 


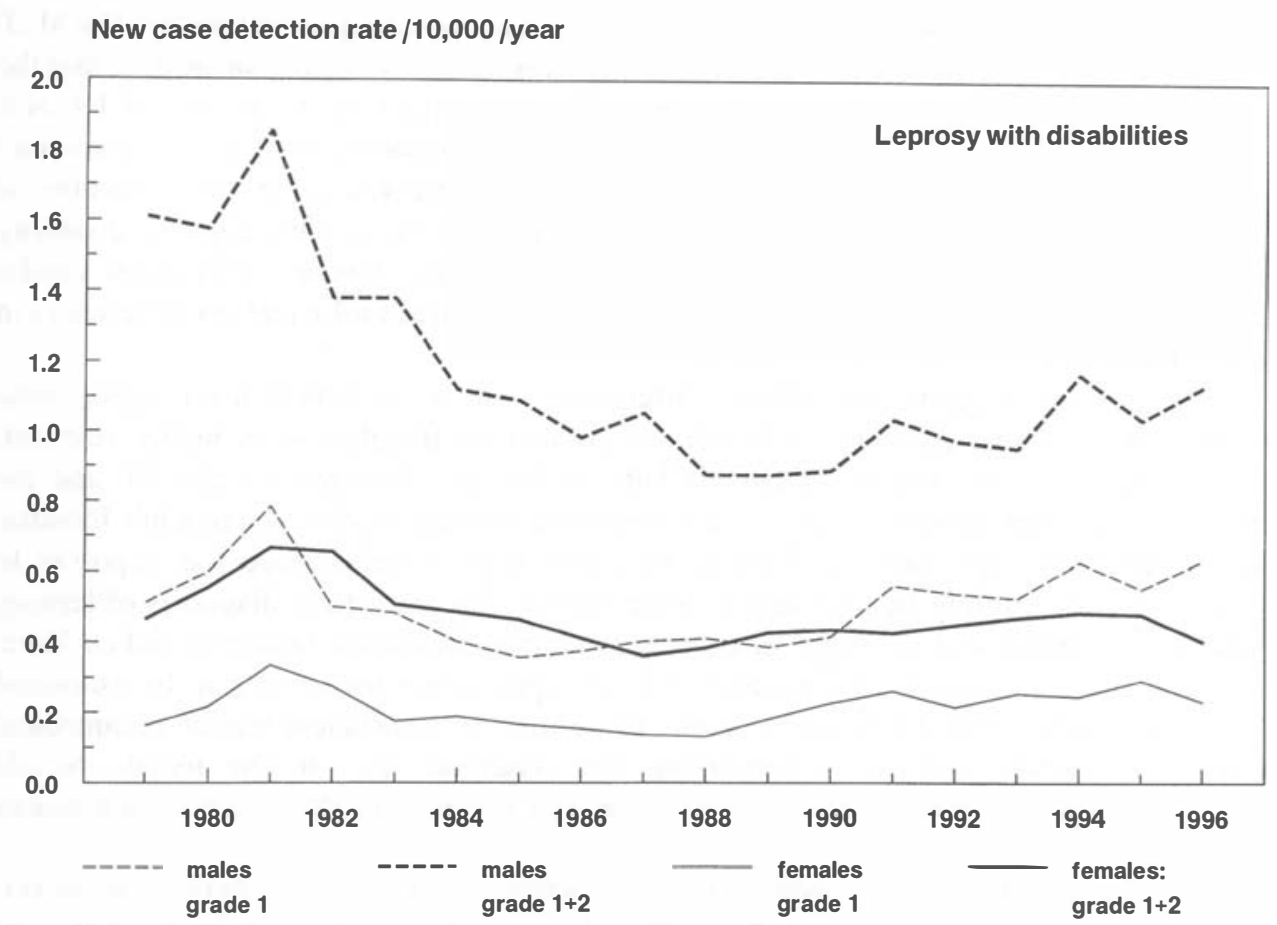

Figure 8. New case detection rate of leprosy patients with disability (grade 1 and grade $1+2$ ) in the DBLM project area per 10,000 general population from 1979 to 1996 , according to sex.

\section{Discussion}

In the 18-year study period the findings at numerator level are largely determined by operational factors in leprosy control activities. Initially, there were few clinics, distributed unevenly over a large area. The number of newly detected cases increased with the expansion of the programme in the last 6-year period. Over-representation of MB leprosy cases in the initial stages was primarily caused by a backlog of easily identifiable leprosy patients, with high deformity rates. Over the last 6-year period, increasing numbers of patients (mainly PB) were detected earlier. The reduction of grade 2 disability also reflects the intensification of case finding. This intensification reduced delays in diagnosis, so preventing patients developing grade 2 disability before treatment.

Caution is warranted in interpreting the declines in $\mathrm{MB}$ proportion and proportion with disability in newly detected cases. The NCDR for MB leprosy has also declined over the study period, especially in males. An impact of this decline on the transmission of leprosy in terms of a reduction in the case detection rate of new cases has not yet been observed. In fact the NCDR increased, due to the intensification of case finding. Furthermore, the decreasing percentages of new cases with disability may falsely suggest that shortly this phenomenon will disappear and therefore no additional efforts are needed to detect and treat nerve function impairment and maintain prevention of disability (POD) programmes. As can be seen in Figure 8, the NCDR of leprosy patients with nerve function impairment and disabilities has not decreased from 1988 onwards, and even shows a clear increase for males. 
This study reveals important gender differences in both detection and disability. The $\mathrm{M}: \mathrm{F}$ ratio in new case detection decreased considerably over the study period, suggesting that the chance of females being detected has increased. To what extent the $\mathrm{M}: \mathrm{F}$ ratio of 1.4 over 1994 to 1996 reflects a gender difference in biological predisposition of males to contract leprosy, exposure-related differences, or operational shortcomings in the detection of females, is still unclear. The observed difference in proportion with grade 2 disability among new cases at the end of the study period (males: $10 \%$, females: $4 \%$ ) raises similar questions; perhaps earlier detection occurs in females, as well as biological sex differences in the incidence of nerve function impairment.

Additional information on gender differences can be obtained from age-specific NCDRs. These reveal that the socio-cultural context of Bangladesh is highly relevant. The interruption in the rise in female NCDRs in the ages between 15 and 30, and the absence of this interruption in males, is an important finding. Maybe young adult females, who in Bangladesh are more confined to their homes than males, have less exposure to leprosy. Also, they might be reluctant to come forward for fear of the diagnosis of leprosy affecting their chances of marriage, or causing separation from their husbands and children. In the Bangladeshi sociocultural context, it is not appropriate for women to be examined by men, and particularly for young women. There may be insufficient female paramedical workers to examine women. Summarizing, the observed 'dip' in the female NCDR probably does not have a biological explanation, but arises from the social circumstances in Bangladesh.

Undoubtedly, females have gained from the intensification of leprosy control. However, more effort is needed to understand the underlying incidence patterns in females, and methods should be developed and tested to reach out to females in the ages range 15-30 years. However, the decrease in $\mathrm{M}: \mathrm{F}$ ratio and the low proportion grade 2 disability in new female cases $(4 \%)$ at the end of the study period are positive signs. However, the higher proportion of grade 2 disability in males $(10 \%)$ does not indicate that females fare better. In the DBLM area, it was shown that the social stigma for females with leprosy disabilities (including skin lesions) is higher than in males. ${ }^{9}$

It has been pointed out that under comprehensive and consistent leprosy control conditions, NCDRs are the nearest approximation of true incidence rates. ${ }^{10}$ NCDRs are therefore a vital indicator for understanding the epidemiology of leprosy. Obviously, control conditions over time in the DBLM programme diverge from this ideal. Nevertheless, this study demonstrates that the application of general population statistics is essential for understanding the dynamics in leprosy control programmes in non-ideal circumstances. Calculation of NCDRs helps to identify population groups that are not benefiting sufficiently from the services provided, and to clarify the dynamics in control programmes and the future trends and programme requirements.

\section{Acknowledgements}

The dedicated work of the Danish Bangladesh Leprosy Mission staff is gratefully acknowledged. Their consistent and skilful performance in often-difficult circumstances has resulted in a model leprosy control programme in a large, remote leprosy endemic area of Bangladesh. Their meticulous record keeping has made it possible to provide this review. 


\section{References}

1 le Grand A. Woman and leprosy: a review. Lepr Rev, 1997; 68: 203-211.

2 Richardus JH, Croft RP. Estimating the size of the leprosy problem: the Bangladesh experience. Lepr Rev, 1995; 66: $158-164$.

3 Chemotherapy of leprosy for control programmes. World Health Organ Tech Rep Ser, 1982; 675: 1-33.

${ }^{4}$ WHO Expert Committee on Leprosy. World Health Organ Tech Rep Ser, 1988; 768: 1-51.

5 Technical guide and operational manual for leprosy control in Bangladesh. TB and Leprosy control services, Government of Bangladesh, Dhala, 1995.

${ }^{6}$ Bangladesh Population Census 1981-National Series. Analytical findings and national tables. Bangladesh Bureau of Statistics, Dhaka, 1984.

7 Bangladesh Population Census 1981-National Series. Volume 1: analytical report. Bangladesh Bureau of Statistics, Dhaka, 1994.

8 Statistical Yearbook of Bangladesh. Dhaka 1995. Bangladesh Bureau of Statistics, Dhaka, 1996.

9 Wilson-Moore M, Richardus JH. Leprosy disability as a physical manif estation of cultural prejudice. Unpublished data.

10 Meima A, Gupte M, Oostmarssen van G, Habbema J. Trends in leprosy case detection rates. Int J Le pr, 1997; 65: 305-319. 